\title{
Non-volatile SRAM memory cells based on ReRAM technology
}

\author{
Hussein Bazzi ${ }^{1,2} \cdot$ Adnan Harb $^{3}$ (1) $\cdot$ Hassen Aziza ${ }^{2} \cdot$ Mathieu Moreau²
}

Received: 9 January 2019 / Accepted: 28 July 2020 / Published online: 8 August 2020

(c) Springer Nature Switzerland AG 2020

\begin{abstract}
Static Random-Access Memories (SRAMs) are very common in today's chip industry due to their speed and power consumption but are classified as volatile memories. Non-volatile SRAMs (nvSRAMs) combine SRAM features with nonvolatility. This combination has the advantage to retain data after power off or in the case of power failure, enabling energy-efficient and reliable systems under frequent power-off conditions. In this work, several nvSRAMs architectures based on Oxide Random-Access Memory (OxRAM) technology are presented and compared. OxRAMs are non-volatile memories considered as a subset of Resistive RAM (ReRAM) technology.
\end{abstract}

Keywords 1T1R ReRAM · Static Random-Access Memory (SRAM) · Non-volatile Static Random-Access Memory (nvSRAM) · Power consumption

\section{Introduction}

The rapid growth in the field of portable electronic devices has been driven by integrated circuits continuous decrease in power consumption and cost. In this context, emerging memories and specifically Non-Volatile Memories (NVM) based on new materials and technologies have flourished [1]. The promising flow of NVM technology is expected to be the pioneer technology for the upcoming years [2]. In the recent years, the interest shifted from Flash memories to alternative NVM technologies based on new materials. These technologies show signs of future success towards enhancing the memory performance and increasing the capability of scaling [3]. Among the different alternative memory technologies, Resistive RAM based on metal oxides known as Oxide Random-Access Memory (OxRAM) have attracted a lot of attention [4]. Indeed, OxRAM devices feature faster READ/WRITE operations, better energy conservation and high endurance than classical Flash memories [5].
Traditional Static Random-Access Memories (SRAMs) are volatile, which is a major handicap regarding powerdown operation where non-volatile memory is needed. OxRAM can be an integral part of the well-known SRAM. This combination called non-volatile SRAM (nvSRAM) integrates both structures in a single cell [6]. nvSRAM offers a direct bit-bit connection to guarantee a fast switching speed and a high rate of data transfer [7]. This concept allows the structure to achieve genuine data retention and low power consumption with small area. Moreover, the non-volatile capability is integrated on the BackEnd-OfLine (BEOL) [8]. Classical low power techniques including clock gating and power gating are commonly used for MCUs power reduction. Clock gating technique is used for reducing dynamic power by controlling switching activities on the clock path [9]. In the case of power gating, certain areas of the chip are idle and other parts are activated only for certain operations [10]. In this context, emerging Non-Volatile Memory (NVM) devices can act as key enablers in the development of ultra-low power (ULP) MCUs [11]. This development opens new area of improvements

\footnotetext{
$\triangle$ Adnan Harb, adnan.harb@b-iu.edu.lb | ${ }^{1}$ Department of Electrical and Electronics Engineering, Lebanese International University, Beirut, Lebanon. ${ }^{2}$ IM2NP-UMR CNRS 7334, Aix-Marseille University, Marseille, France. ${ }^{3}$ Department of Electrical and Electronics Engineering, International University of Beirut, Beirut, Lebanon.
} 
in the ability of computing and energy conservation of existing systems including loT applications [11]. The rapid expansion of emerging memories reached SRAMs technology that really needs a push to overcome serious problems including the reduction of the power supply and in the transistor size, leading to an increase of leakage currents [12].

Proposed nvSRAM designs are implemented in a high voltage 130-nm technology from STMicroelectronics. In section II, the basic OxRAM model used in this work is presented along with the 1T1R OxRAM cell considered in this study. Section III proposes a detailed overview of OxRAMbased nvSRAM structures, with a deep looking on the ability of the nvSRAM to STORE and RESTORE data. In section IV, a discussion and a comparison of the different nvSRAM cells are proposed. At the end of this paper, the conclusion summarizes all the concepts of previous sections.

\section{ReRAM technology}

\subsection{OxRAM model}

The OxRAM model approach used in this work is based on the formation and destruction of oxygen vacancies by the induced electric field inside the insulator layer [5]. In this model, SET and RESET (operations used to switch between high and low resistive stats) are constantly managed by a distinct equation where the radius of the conductive filament controls the resistance [13]. This equation is given as:

$$
\begin{aligned}
\frac{d r_{C F}}{d t}= & \left(r_{C F \max }-r_{C F}\right) \cdot 10^{\beta_{\text {Redox }}} \cdot e^{-\frac{E a-q \cdot x_{\text {red }} \cdot v_{\text {cell }}}{k_{b} \cdot T}} \\
& -r_{C F} \cdot 10^{\beta_{\text {Redox }}} \cdot e^{-\frac{E a+q \cdot \alpha_{0 X} \cdot v_{\text {cell }}}{k_{b} \cdot T}}
\end{aligned}
$$

where $\beta_{\text {RedOx }}$ is the nominal oxide reduction rate, $E_{a}$ is the activation energy, $a_{\text {red }}$ and $a_{o x}$ are the transfer coefficients (ranging between 0 and 1 ), $\mathrm{k}_{\mathrm{b}}$ is the Boltzmann constant, $r_{C F m a x}$ is the maximal size of the conductive filament radius, $\mathrm{T}$ is the temperature and $\mathrm{V}_{\text {cell }}$ the voltage across the cell.

Furthermore, some assumptions are considered in the model including a uniform electric field and radius of the conductive filaments. Also, the acceleration of the oxide reactions is triggered by the temperature. Lastly, two components are included in the OxRAM total current, which are the current linked to the conductive species $\left(\mathrm{I}_{\mathrm{CF}}\right)$ and the current related to the conduction through the oxide $\left(\mathrm{I}_{\mathrm{OX}}\right)$. The two equations for $\mathrm{I}_{\mathrm{CF}}$ and $\mathrm{I}_{\mathrm{OX}}$ are formulated as follow:

$I_{C F}=\frac{V_{C e l l}}{L_{x}} \cdot\left(\pi \cdot r_{C F}^{2} \cdot\left(\sigma_{C F}-\sigma_{O X}\right)+\pi \cdot r_{C F \max }^{2} \cdot \sigma_{O X}\right)$
$I_{O X}=A_{H R S} \cdot S_{C e l l}\left(\frac{V_{C e l l}}{L_{x}}\right)^{\beta_{H R S}}$

where $L x$ is the oxide thickness, $\mathrm{S}_{\mathrm{Cell}}$ is the total area of the device, $\sigma_{\mathrm{OX}}$ the oxidation rate and $\sigma_{\mathrm{CF}}$ the reduction rate. The two parameters $A_{H R S}$ and $\beta_{H R S}$ alongside the power law between the bias applied and the cell current are considered, thus $\mathrm{I}_{\mathrm{Ox}}$ trap assisted current can be taken into account. Finally, the total current passing through the cell is expressed as follow:

$I_{\text {Cell }}=I_{C F}+I_{O X}$

This model is calibrated on silicon (130 nm technology from STM) with no convergence issues when used in combination with CMOS technology. Also, it is considered as one of the few model taking into account the FORMING operation, which is a crucial factor when targeting the fabrication of the proposed devices. Moreover, the $130 \mathrm{~nm}$ technology provides High Voltage (HV) transistors needed for the FORMING operation. The need of a HV option (around $3 \mathrm{~V}$ ) prevents the use of more advanced technology nodes. Indeed, a prospective study in more advanced technology node is pertinent as the resistive element can be integrated in the BEOL between metal lines. The work in [14] demonstrates a 16 Gb ReRAM designed in a $27 \mathrm{~nm}$ node, with a $1 \mathrm{~GB} / \mathrm{s}$ DDR interface and an 8-bank concurrent DRAM-like core architecture.

\subsection{T1R structure}

The OxRAM cell consists of three layers, including the insulator layer (switching layer) working as the storage medium which is located between two metallic electrodes (top electrode TE and bottom electrode BE). This Metal-Insulator-Metal (MIM) structure is integrated over a Metal 4 copper layer $(\mathrm{Cu})$ as shown in Fig. 1a. First, the TiN $\mathrm{BE}$ is deposited. Then, a $10 \mathrm{~nm}-\mathrm{HfO}_{2} / 10 \mathrm{~nm}-\mathrm{Ti} / \mathrm{TiN}$ stack is added to form a capacitor-like structure [15].

One of the common selector used with ReRAM devices is the CMOS transistor. In this context, the 1 transistor-1 resistor (1T1R) structure has been widely used, where the OxRAM cell is connected on the top of the transistor as shown in Fig. $1 \mathrm{~b}$. In addition, the transistor controls the compliance current passing through the OxRAM cell during programming operations to prevent memory cell damage.

Figure $2 \mathrm{a}$, b present 1T1R OxRAM I-V characteristics in linear scale and logarithmic respectively extracted from actual OxRAM devices [16]. The principle operation of OxRAM based memory cells consists of several stages [17]. The first step is the Forming operation which is executed 


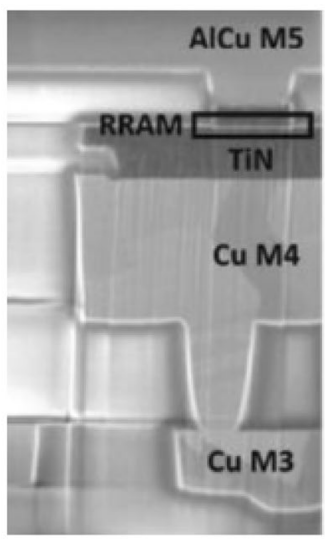

(a)

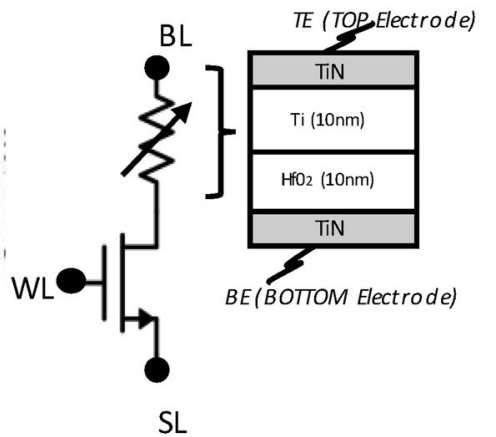

(b)
Fig. 1 a MIM structure and b Basic 1T-1R OxRAM cell

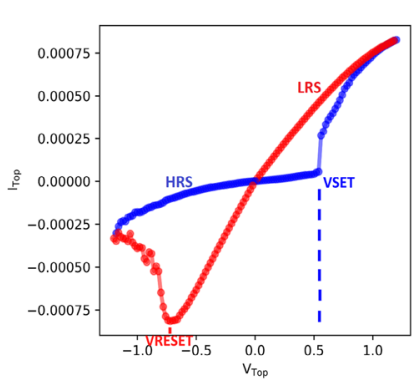

(a)

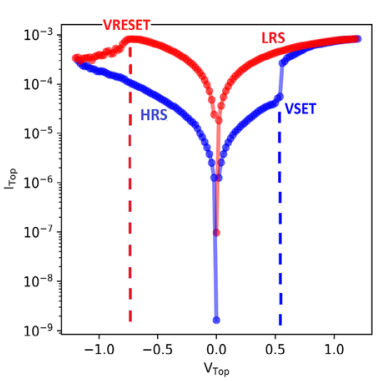

(b)
Fig. 2 I-V characteristic of the OxRAM model in $\mathbf{a}$ linear and $\mathbf{b}$ log scale

once in the life of OxRAM cell, where a high voltage is induced to switch from High Resistance State (HRS, or pristine state) to Low Resistance State (LRS) [18]. After Forming, the cell can be switched between HRS and LRS by applying specific voltages across the electrodes of the OxRAM cell (i.e. $V_{\text {SET }}$ and $V_{\text {RESET }}$ ) [19]. Based on the linear curve presented in Fig. 2a, $V_{S E T}$ value needed to switch to LRS state is equal to $0.57 \mathrm{~V}$, while the $\mathrm{V}_{\text {RESET }}$ value required to switch back to HRS state is equal to $-0.7 \mathrm{~V}$. Note that the log curve is the classical representation of the OxRAM I-V hysteresis as it amplifies low current values. Regarding reliability, as demonstrated in [20], SET/RESET endurance was evaluated up to $10^{8}$ cycles showing that the oxide-based technology is in agreement with SRAM operation.

The behavior of a ReRAM is subject to various deviations, somewhat different from the ones affecting traditional CMOS components. The realization of ReRAM is affected by the manufacturing process. Process variations such as variations in size or ion concentration, environmental conditions (e.g., ambient temperature) or circuit parameters (e.g., variations in power supply) $[21,22]$ may cause the deviation of the actual electrical behavior of ReRAM from the original design and result. Therefore, it is very important to understand and characterize the impact of process variations on the electrical behaviors of the ReRAM and its implication to the circuit design. Moreover, as the process technology scales, device parameter fluctuations induced by process variations are such as lineedge roughness (LERs) caused by uncertainties in the process of lithography and etching [23], oxide thickness fluctuations (OTFs) caused during sputtering or atomic layer deposition, and random discrete doping (RDDs). The effect of process variations on resistance is similar for both HRS and LRS resistances.

Note that for most nvSRAM cells architectures, extra reliability is provided by the symmetry of the memory cell architectures. Indeed, since the cell is differential, the 2 OxRAMs are affected the same way by variability. Also, as the OxRAM cells are disconnected from the SRAM core cell during the read operation, they are not involved in the read operation, so the read operation is not directly impacted by OxRAM variability.

Concerning the degradation of the OxRAM devices, it is possible to reach an outstanding endurance of twenty billion cycles with the technology considered in the paper [24], which motivates the use of OxRAMs for nvSRAM applications. Figure 3 presents the evolution of the ON/ OFF (LRS/HRS) resistance ratio during cycling for 2 OxRAM devices.

Recently, the retention mechanism of ReRAM has attracted considerable attention. At lower temperatures, the conductivity mechanism follows $T^{-1 / 4}$, describing variable range hopping conductivity. On the other hand, the conductivity mechanism follows $\mathrm{T}^{-1}$ at higher temperatures, describing fixed range hopping conductivity [25]. In this context, several works have exploited the data retention of ReRAM with different temperature [26]. In [25], a ReRAM based memory with long-term retention exceeding 10 years at $85^{\circ} \mathrm{C}$ is successfully demonstrated. Figure 4 . represents the time to retention failure with respect to temperature $(1000 / T)$.
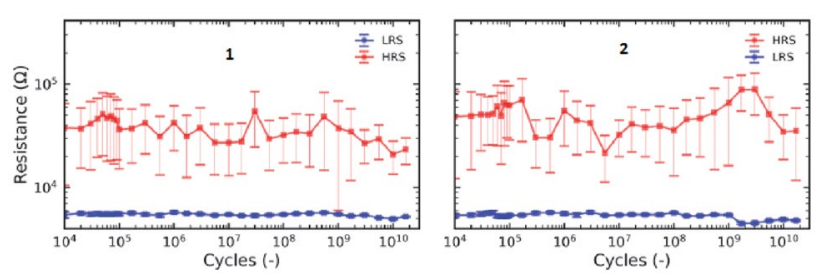

Fig. 3 SET/RESET endurance evaluated for 2 OxRAM devices [24] 


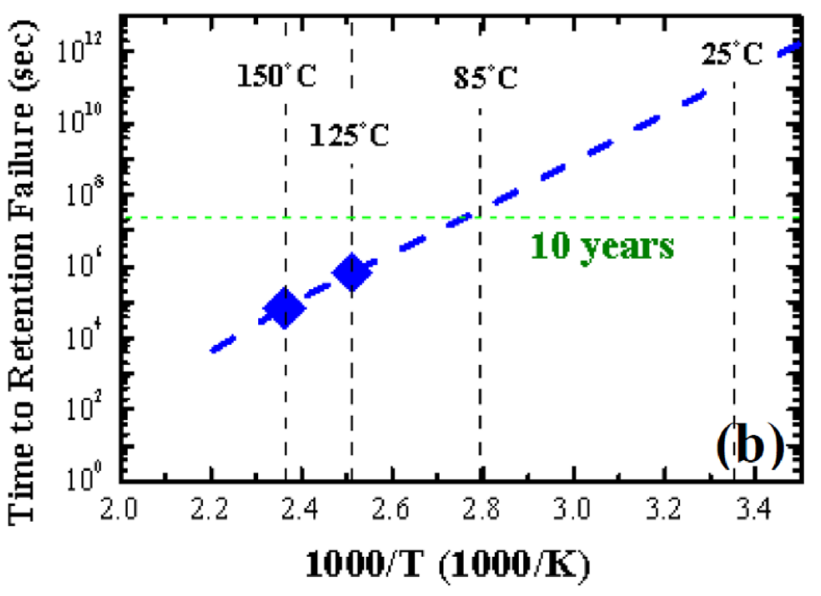

Fig. 4 Time to retention failure [25]

\section{1T1R OxRAM cell simulation}

The OxRAM model used for simulations is a compact model calibrated on silicon well suited to simultaneously describe SET and RESET operations [27]. Figure 5 presents timing waveform of the $1 \mathrm{~T} 1 \mathrm{R}$ cell presented in Fig. 1 b during FORMING, RESET \& SET operations. The duration of these 3 operations are $13 \mu \mathrm{s}, 4 \mu \mathrm{s}$ and $1 \mu \mathrm{s}$ respectively.

$\mathrm{V}(\mathrm{TE}, \mathrm{BE})$ represents the voltage across the OxRAM device. The voltage at the gate of the transistor $\mathrm{V}(\mathrm{WL})$ is kept high. During FORMING, the voltage at the top electrode $\mathrm{V}(\mathrm{BL})$ is also set high $(3.5 \mathrm{~V})$, and the voltage at the bottom electrode $\mathrm{V}(\mathrm{SL})$ is $0 \mathrm{~V}$. After FORMING, OxRAM device is RESET with $\mathrm{V}(\mathrm{BL})=0 \mathrm{~V}$ and $\mathrm{V}(\mathrm{SL})=2.7 \mathrm{~V}$. During the SET operation, $\mathrm{V}(\mathrm{BL})$ is set to $1.8 \mathrm{~V}$.

\section{Non-volatile SRAM}

\subsection{NVSRAM memory cells}

As already mentioned in the introduction, one of the solution to reduce/eliminate standby power in SRAM cells since it to turn the traditional volatile SRAM non-volatile. The addition of this feature is important to eliminate the standby leakage currents. Indeed, the memory can operate in a conventional way in active mode and can be powered OFF in standby mode to prevent standby power consumption without losing its data. Therefore, NVSRAM and SRAM cells have similar data retention time when working in the conventional way (HOLD mode). In the non-volatile mode, NVSRAM cells follows the data retention time of the ReRAM devices used.

In the next sections, five different types of nvSRAMs are presented. These architectures include Majumdar's 4T2R cell [28], Wei's 7T1R cell [29], Turkyilmaz's 8T2R cell [30], Sheu's 7T2R cell [31], and Chiu's 8T2R cell [32].
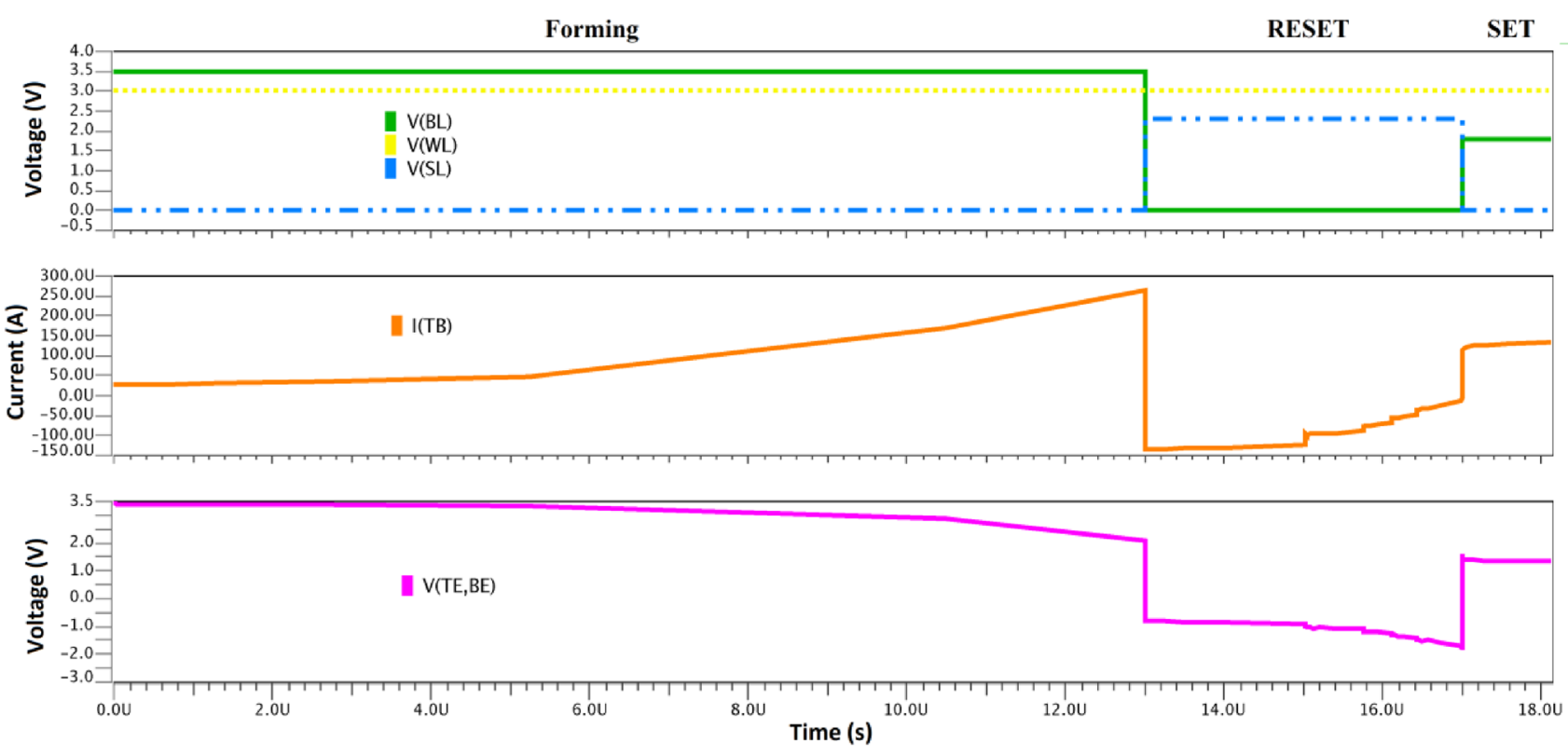

Fig. 5 1T1R timing waveforms 


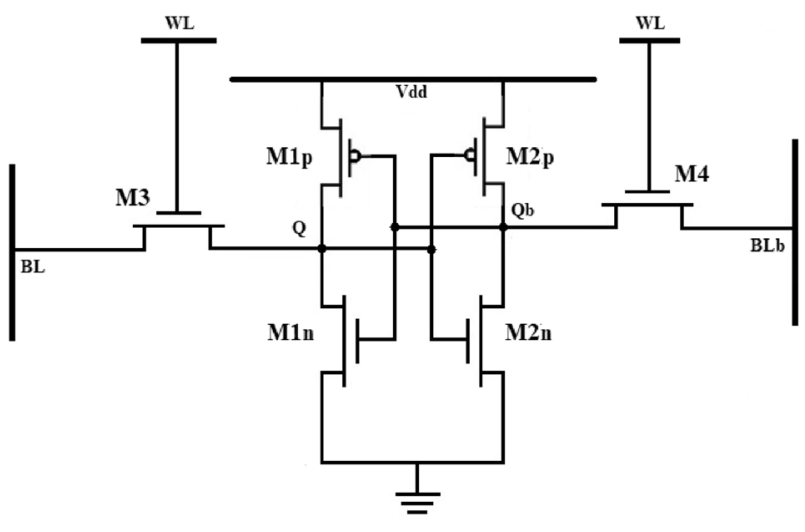

Fig. 6 6T SRAM Cell

Each nvSRAM cell is based on the conventional SRAM structure. The conventional SRAM structure follows the cross coupled inverters structure presented in Fig. 6 . The 3 main operations in a SRAM are HOLD, READ and WRITE. At an SRAM memory array level, rows are called Word Lines (WLs) and columns are called Bit Lines (BLs). WLs are connected to the gates of the select transistors (M5 and M6).

WRITE and READ operations in the memory cells are accomplished through the bit lines. During a HOLD operation, the $W L$ is deactivated to disconnect $B L$ and $B L b$ from the SRAM cell. As a result, data is held in the latch structure on nodes $Q$ and $Q b$. When WL is activated, READ and WRITE operations can be executed.

\section{4T2R cell [28]}

The 4T2R nvSRAM cell [28] is presented in Fig. 7. It consists of 2 OxRAMs, 4 Transistors, where M3 and M4 act as access transistors connecting the bit lines to $\mathrm{Q}$ and $\mathrm{Qb}$ nodes. FORMING operation is mandatory before operating the nvSRAM. Note that OxRAM devices R1 and R2 need a high voltage on the top electrodes to be formed. Note that a STORE operation (SET or RESET operation of the OxRAM cell) is associated with a WRITE operation. During WRITE/STORE, the bit lines are connected to the nodes $Q$ and $Q b$. The potential difference between TE $(Q$ or $\mathrm{Qb}$ ) and $\mathrm{BE}$ ( $\mathrm{PL}$ line) of the OxRAM element will either induce a SET (ON state) or RESET (OFF state) operation of the cell. After WRITE/STORE operation the cell data

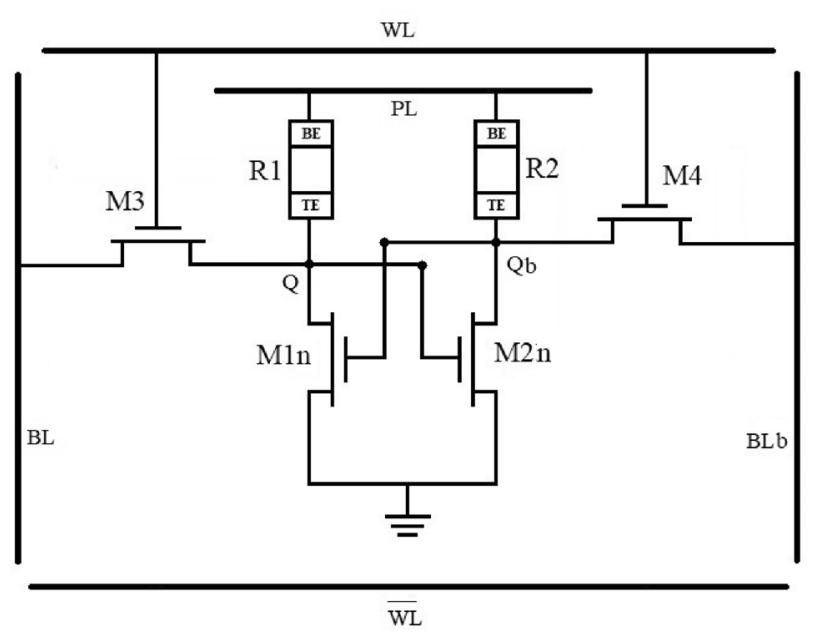

Fig. 7 4T2R nvSRAM Cell

remains unchanged as well as the cell resistances provided that no sufficient voltage potential difference is seen across the OxRAM cells. After power shutdown, the nvSRAM last state can be restored as the data is saved in a non-volatile resistance value. The restored nvSRAM value can be checked during a READ operation after powering on the cell. PL line is connected to $V_{D D}$ and a certain amount of current will pass through the OxRAM devices depending on their resistance values.

Figure 8 presents timing waveforms of the 4T2R Cell. During FORMING, BL and BLb are high and $W L$ and $P L$ low. After FORMING, R1 is RESET while R2 stays in LRS state with BLb and PL high and BL low. The next following 2 steps are Pre-charge and READ to check the memory state. $B L$ and $B L b$ are pre-charged to $V_{D D} / 2$ and $P L$ is set to $1 \mathrm{~V}$ to not affect $R 1$ and $R 2$ resistance values during the next read operation.

During READ, WL is turned ON thus the data on the nodes can transmitted to the bit lines to reach the reading circuitry. After that, R1 device is SET and R2 is RESET consecutively following the same programming scheme of the previous STORE operation. Since the nVSRAM is non-volatile, the memory cell is powered down to test the ability of the cell to recover the data. The shutdown is followed by a RESTORE operation where a $1 \mathrm{~V}$ voltage is applied to PL to get the data back to the storage nodes. After RESTORE, a Pre-charge and READ are performed to check $Q$ and $Q b$ values. 

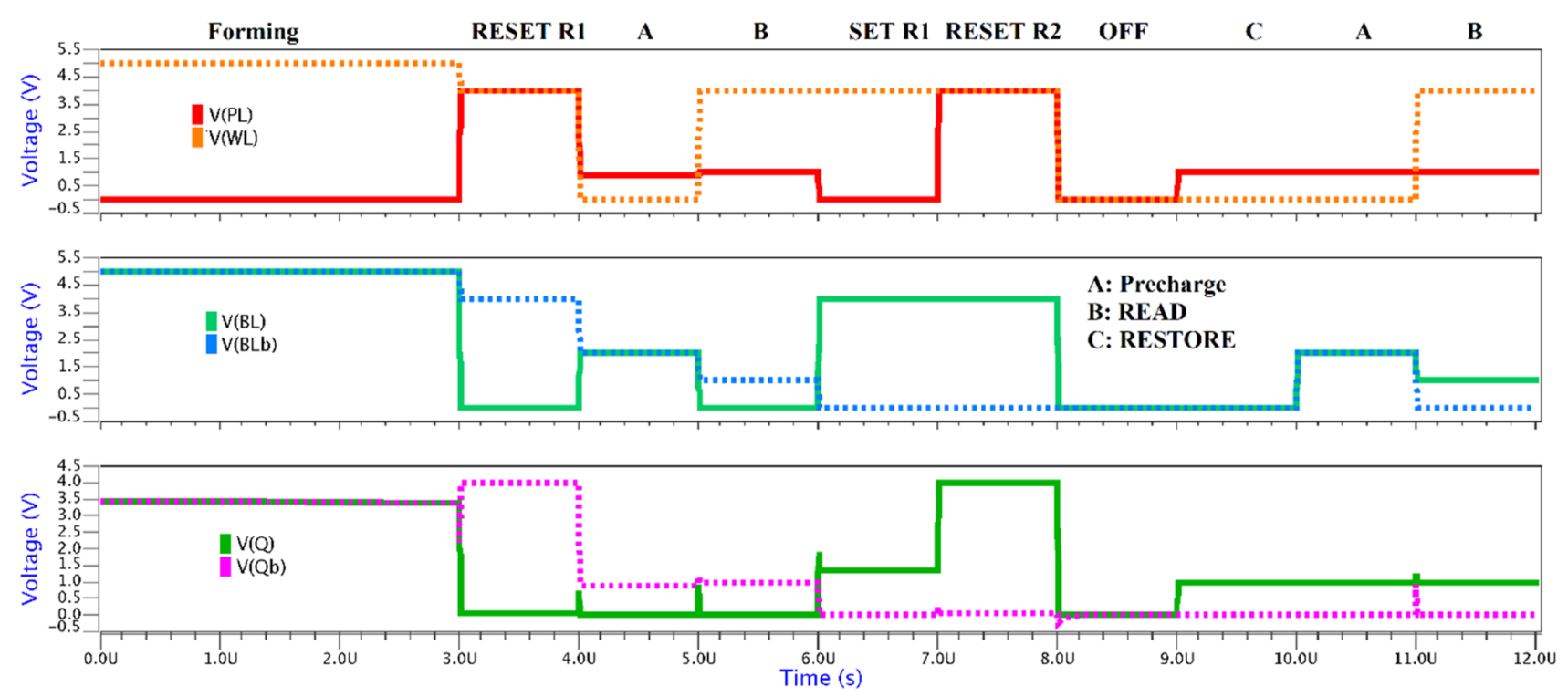

Fig. 8 4T2R timing waveforms

\section{7T1R cell [29]}

The 7T1R nvSRAM cell [29] is presented in Fig. 9. The structure of this cell consists of 1 OxRAM, 7 Transistors, including 2 CMOS inverters and 2 access transistors. M5 access transistor acts as switch controlling the STORE operation. In this configuration, one OxRAM device is directly connected to the memory nodes $Q$ which is used to store logic states " 0 " and " 1 ". The resistance of the OxRAM switches between HRS and LRS depending on the data stored at nodes $\mathrm{Q}$ and $\mathrm{Qb}$. As the previous structure, FORMING is executed at the beginning but only through $\mathrm{BL}$ since there is only 1 OxRAM device.

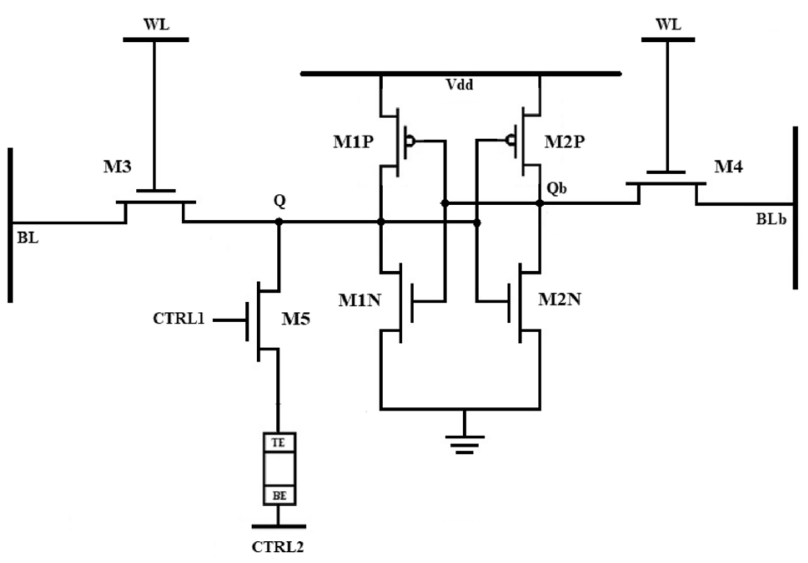

Fig. 9 7T1R nvSRAM Cell
During the STORE operation, the data on the bit line is transferred to the node $Q$, while CTRL1 is high and CTRL2 is grounded. For instance, if the node $Q$ data is "1", the potential difference across the OxRAM changes the resistance from HRS to LRS. This setup is mandatory to program the device depending on node $\mathrm{Q}$ voltage. At the end of this operation, the resistance state of the OxRAM matches the logic states stored at the data nodes ( $Q$ and $Q b)$. Note that before STORE, node $\mathrm{Q}$ is grounded to discharge any voltage left at this node.

During power-down stage, all the voltage sources are shutdown. During RESTORE operation, the current from CTRL2 passes through the OxRAM device depending on the resistive state. For instance, if the OxRAM is in LRS, the node Q stays at "1" and Qb is discharged through the NMOS transistor M2n.

During READ operation, WL and CTRL1 are high, thus the current passes in the OxRAM device depending on its resistance value. Node $\mathrm{Q}$ is connected to $B L$ to sense the memory cell state.

Timing waveforms of the 7T1R cell presented in Fig. 10 differ from the 4T2R cell as WRITE and STORE operations are executed separately. During FORMING, BL is high with WL and CTRL1 set high. RESET is preceded by a WRITE " 0 " operation where node $\mathrm{Q}$ data is stored in the OxRAM device. Note that this the 7T1R architecture can work as a traditional SRAM when M5 is OFF (i.e. independently from the OxRAM cell). BL is high during FORMING and WRITE "1" operation. CTRL1 is high during Forming, SET, RESET and RESTORE operations. The value of the current passing through R1 I(TB) is only considered when CTRL1 (Vctrl) is 

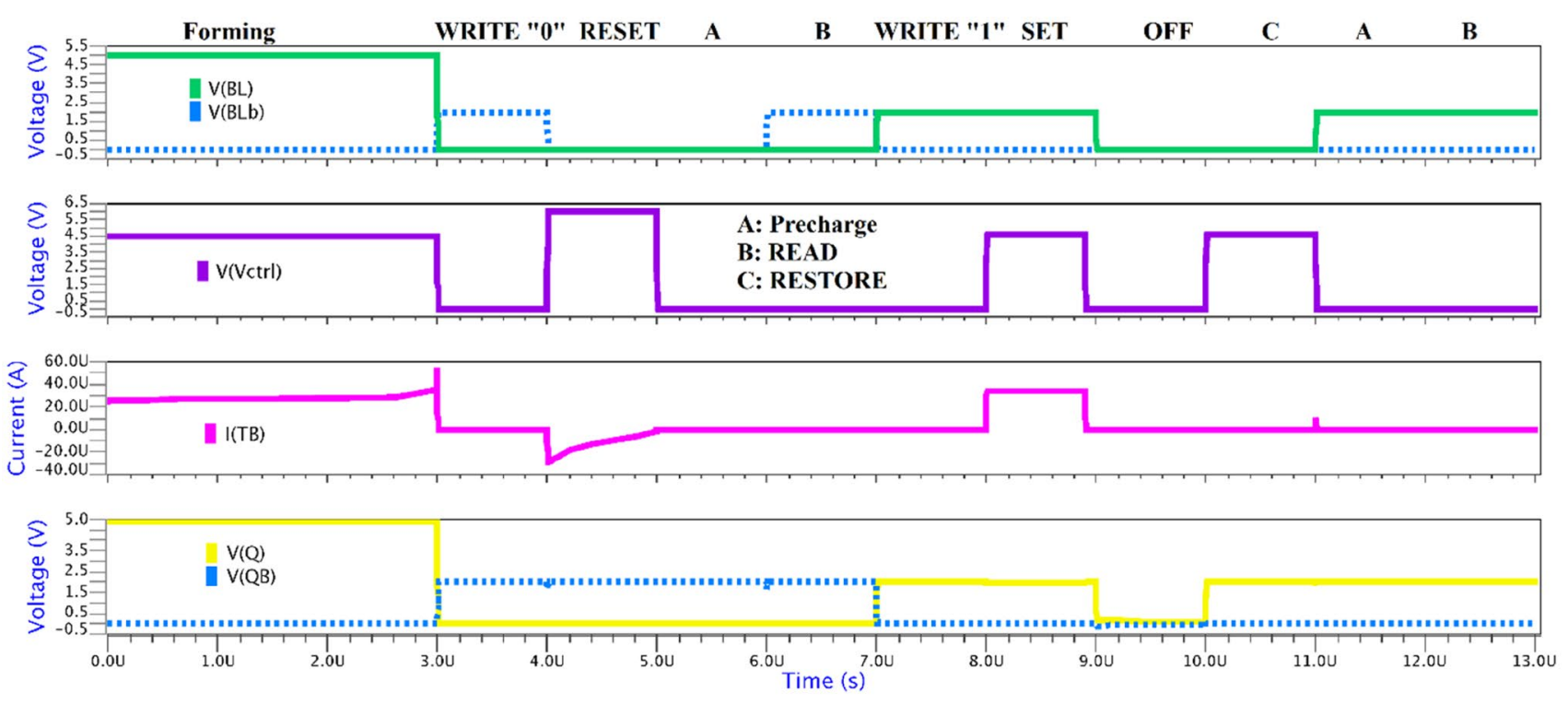

Fig. 10 7T1R timing waveforms

ON, since no current flows in the device when it is OFF. I(TB) increases gradually during FORMING.

RESET operation is performed by applying a high voltage on CTRL1 and CTRL2, while WL is grounded. During this operation, I(TB) decreases to nearly $0 \mathrm{~V}$ since the OxRAM switched to the HRS. On the other hand, during SET I(TB) increases as the OxRAM is in LRS state. In RESTORE, CTRL1 is high and the restore voltage is applied on CTRL2 where the current I(TB) will pass through the OxRAM depending on the resistive state (HRS or LRS). After RESTORE, a Pre-charge and READ are performed to check $\mathrm{Q}$ and $\mathrm{Qb}$ values.

\section{8T2R cell [30]}

The design proposed in Turkyilmaz paper [30] is very similar to 7T1R cell [29], as two OxRAM devices are used instead of one. However, OxRAMs are accessed using two transistors M3 and M4 controlled by CTRL1 signal as shown in Fig. 11. Regarding the operation principle, the same procedure used for the 7T1R structure can be followed but considering 2 OxRAM cells. SET and RESET are performed by activating M5 and M6. In case of SET, CTRL1 is high and CTRL2 is grounded and according to the data stored in the nvSRAM cell, either R1 or R2 is set to LRS. During RESET CTRL2 is SET to $V_{D D}$ and according to the data stored in the nvSRAM cell, either R1 or R2 is set to HRS.

During RESTORE, if $R 1$ is in LRS, node $Q$ is "refreshed" with logic '1'. If $R 1$ is in HRS, node $Q$ is "refreshed" with logic ' 0 '. R2 follows the same procedure.

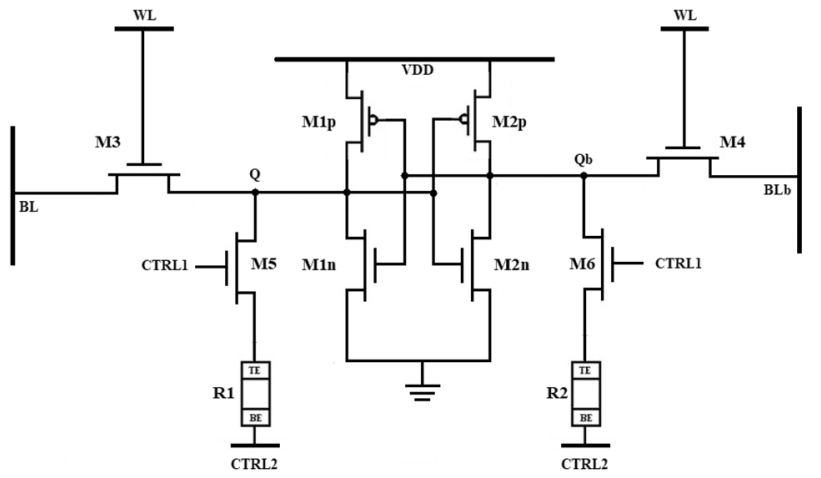

Fig. $118 T 2 R$ nvSRAM Cell

After RESTORE, R1 and R2 are disconnected from the rest of the circuit and the SRAM is not effected by the OxRAM cells (i.e. M5 and M6 isolate the OxRAM from the SRAM core to avoid the degradation of the cell performance in the normal mode operation).

Timing waveforms presented in Fig. 12 are practically the same as the 7T1R memory cell [29], but since the 7T1R design is built using 2 OxRAM devices, 2 FORMING operations are needed. During the FORMING stage, BL is set high to form OxRAM R1 (current I1(TB) increases). Then, BLb is set high to form OxRAM R2 (current 12(TB) increases). After FORMING, the $8 \mathrm{~T} 2 \mathrm{R}$ cell follows the same programming routine as the 7T1 $R$ cell but considering two OxRAM devices instead of one. Currents I1 (TB) and I2(TB) change depending on the executed operation. During RESTORE, CTRL1 is ON and CTRL2 is set to $1 \mathrm{~V}$. During READ operation, $\mathrm{WL}$ and CTRL1 are set high. 

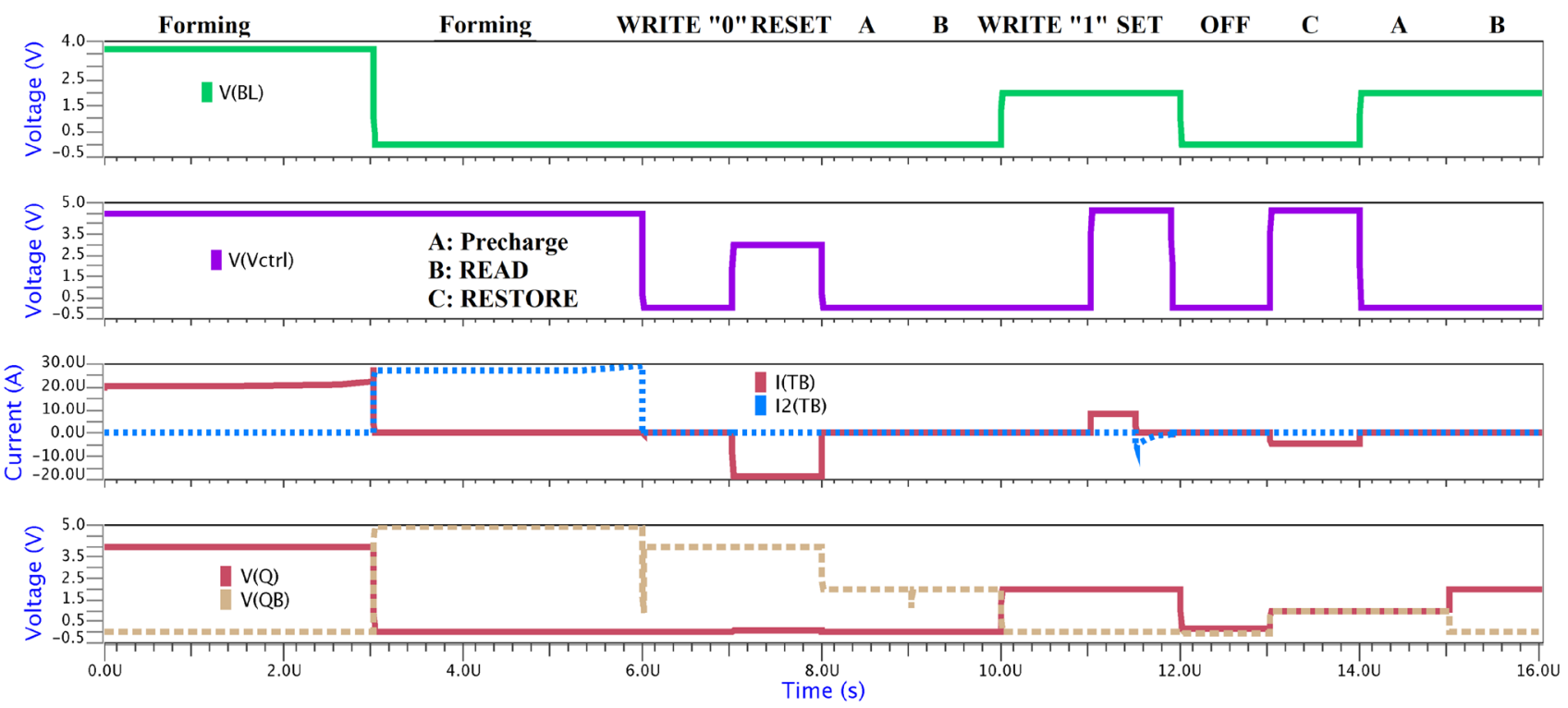

Fig. $128 \mathrm{~T} 2 \mathrm{R}$ timing waveforms

\section{7T2R cell [31]}

The design proposed in Sheu paper [31] is close to $8 \mathrm{~T} 2 \mathrm{R}$ cell, excepted that a single signal SWL controls STORE/ RESTORE operations as presented in Fig. 13. The 8T2R consists of 2 OxRAMs, 7 transistors including 2 CMOS inverters and 3 access transistors. $M_{\text {NSW }}$ transistor controls STORE/ RESTORE operations. Also, OxRAMs bottom electrodes (BE) are connected to $\mathrm{M} 3$ and $\mathrm{M} 4$ which connect the cell to the bit lines.

FORMING operation in the 7T2R cell follows this sequence: WRITE " 0 " on node $Q, R_{L}$ FORMING, WRITE "1"

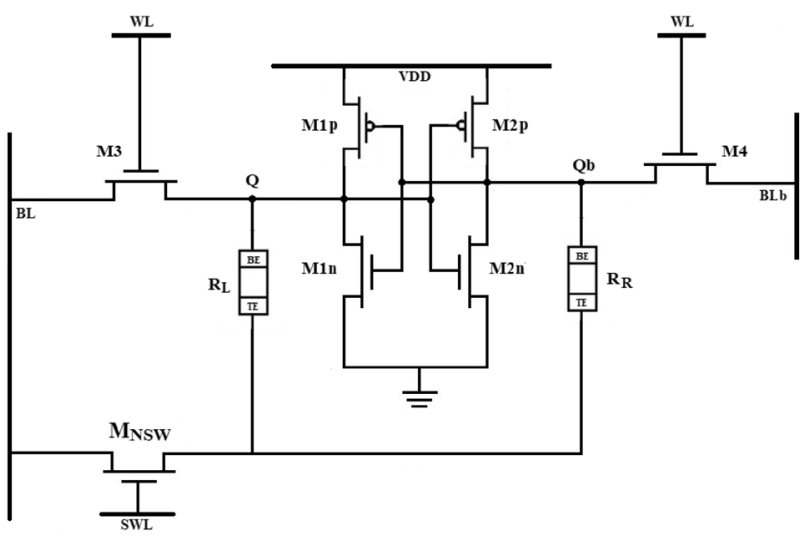

Fig. $137 T 2 R$ nvSRAM Cell on node $Q$, and $R_{R}$ FORMING. Note that FORMING operation is performed through BL for both OxRAMs.

During STORE, OxRAMs resistance changes from LRS and $\mathrm{HRS}$ respectively depending on $\mathrm{Q}$ and $\mathrm{Qb}$ node voltages provided that SWL signal is set high. During READ operation, $W L$ is high and $S W L$ low, disconnecting $R_{L}$ and $\mathrm{R}_{\mathrm{R}}$ from the core SRAM cell.

Timing waveforms are presented in Fig. 14. FORMING operation in 7T2R cell [31] is divided into 2 stages $\left(R_{L}\right.$ and $R_{R}$ FORMING). The first stage starts with WRITE " 0 " with $B L$ and SWL set low and $W L$ set high. Then, $R_{L}$ is formed with $B L$ and SWL set high and WL set low. In the second stage, $R_{R}$ FORMING is achieved starting with WRITE "1" operation with $B L$ and WL set high and SWL set low. Note that $B L, S L$ and $S W L$ have the same programming levels during $R_{L}$ and $R_{R}$ FORMING.

RESET is performed after WRITE "1" operation, where I1(TB) decreases to approximately $0 \mathrm{~V}$ (R1 in HRS state). WL is high and SWL is low during WRITE, and the opposite during RESET operation. WRITE " 0 " operation is needed before SET. WL and SWL follows the same pattern as in (WRITE/RESET) sequence. During programming, WRITE " 0 " on the node $Q$ and "1" on $Q b$ are executed simultaneously. During the STORE operation, BL is high to SET R1 and low to RESET R2. Note that BL is the only bit line participating in this operation. During RESTORE, the data retained at node $Q$ is " 1 " if the OxRAM device is in LRS state and " 0 " if it is in HRS state. During READ operation, WL is set high and SWL is set low. 

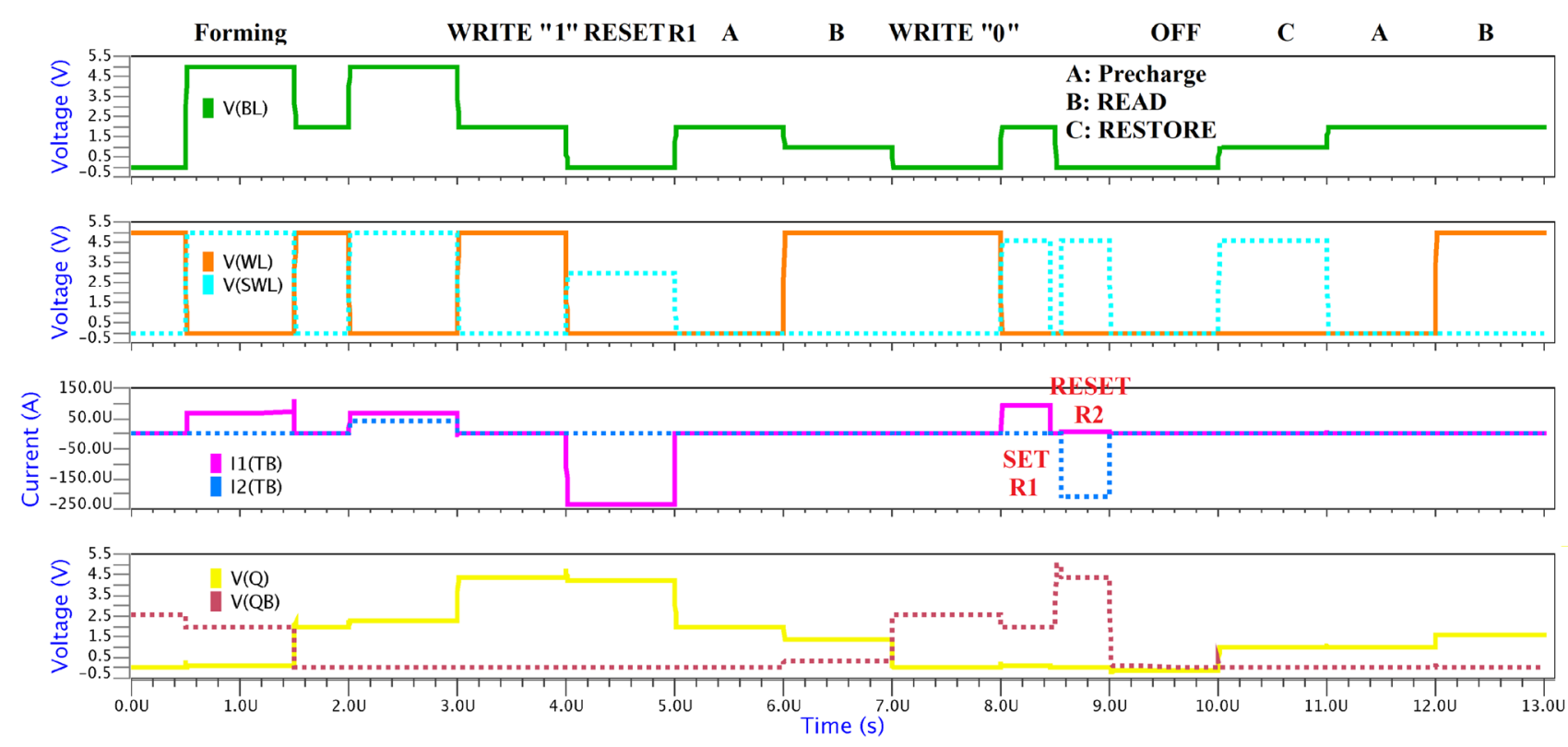

Fig. 14 7T2R timing waveforms

\section{8T2R cell [32]}

The design proposed by Chiu [32] is based on the 7T2R structure presented in Fig. 13 except that the top electrodes of the OxRAM cells are connected to the different bit lines. The 8T2R cell is depicted in Fig. 15.

The proposed nvSRAM cell spares on control signal as M5 and M6 transistors are controlled by BL and

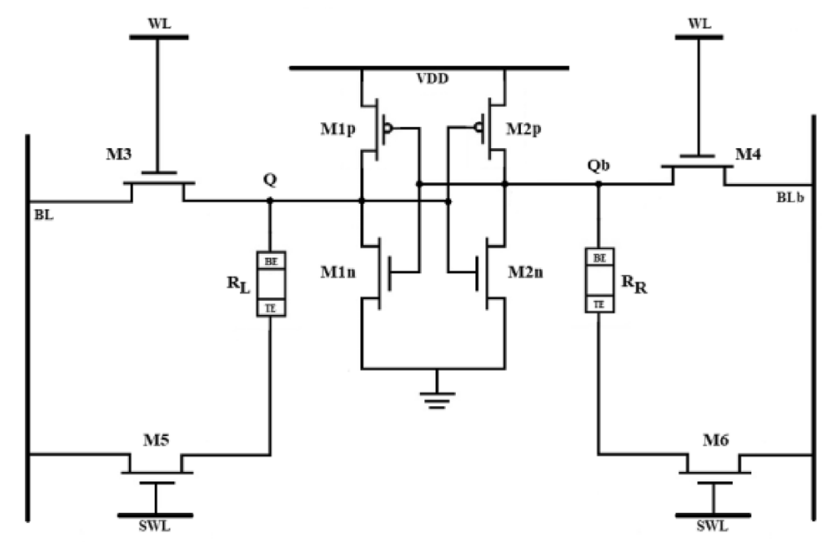

BLb respectively. In this design, FORMING is executed through both bit lines (BL and BLb).

After FORMING, the corresponding bit line is used to STORE (SET/RESET) data on the OxRAM. The switch-line (SWL) is grounded to turn off the selector transistors connected to the bit lines and to prohibit any potential disturbance that affects the stability of the SRAM cell. FORMING operation in this design is performed through the bit lines $B L$ and $B L b$ connected to $R_{L}$ and $R_{R}$ respectively. During RESTORE, data stored on the OxRAM devices are recalled to the nodes ( $\mathrm{Q}$ and $\mathrm{Qb}$ ).

Timing waveforms of this structure are close to that of 7T2R cell [31] as shown in Fig. 16. During FORMING both $\mathrm{BL}$ and $\mathrm{BLb}$ are high to form the 2 OxRAM devices at the same time, thus the currents (I1 (TB and (I2(TB)) increase simultaneously. The currents I1(TB) and I2(TB) will react depending on the operation executed.

BLb is low during WRITE " 1 ", and high during WRITE " 0 ". During RESTORE, SWL is high and WL is low with restore voltage is applied at the bit lines.

Fig. 15 8T2R nvSRAM Cell (CHIU) 

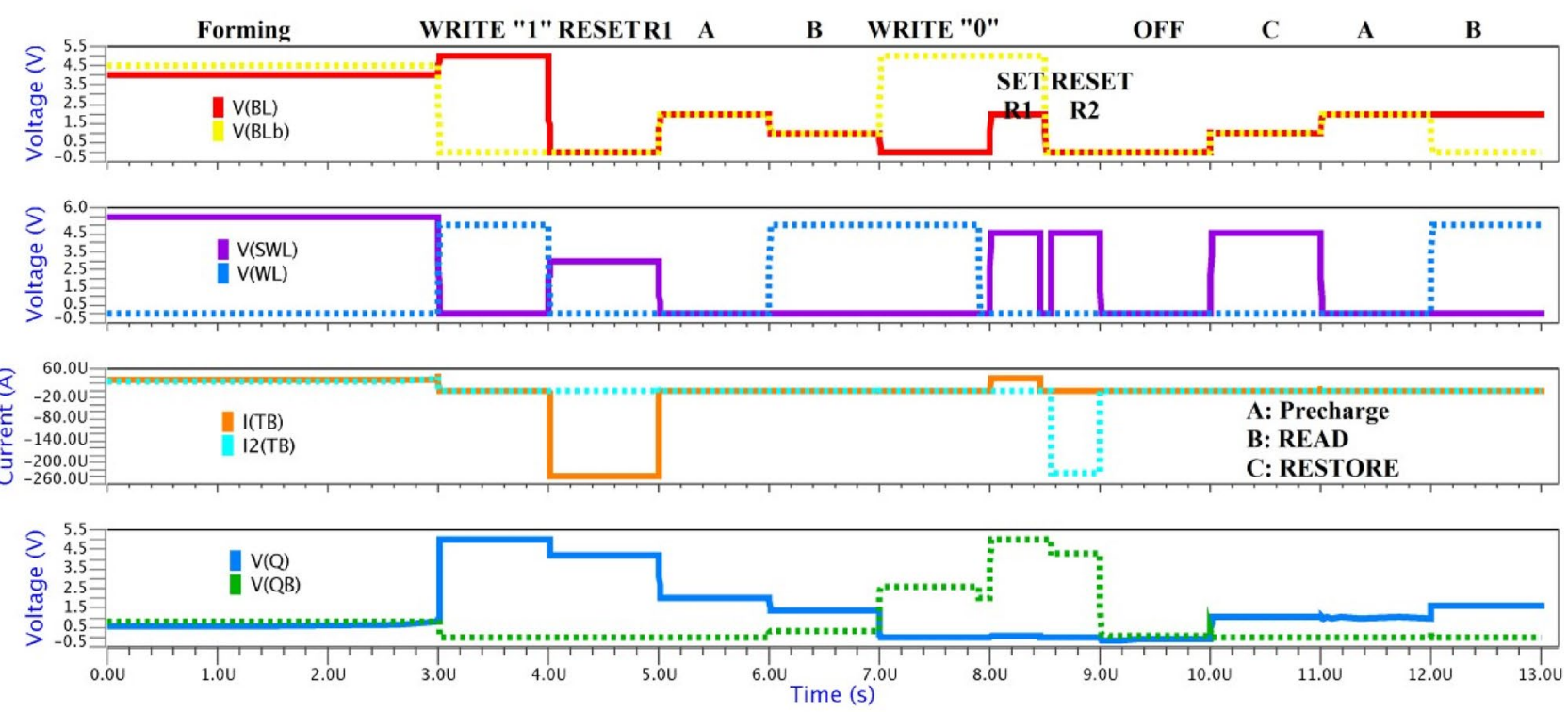

Fig. 16 8T2R timing waveforms (CHIU)

\section{Structure comparison and discussions}

In this section, 5 nvSRAM cells are discussed and compared showing their advantages and limitations. Table 1 presents nvSRAM cells original CMOS and ReRAM technology used to build the structures. Table 2 illustrates advantages/drawbacks of all nvSRAMs in terms of STORE/
RESTORE time, RESTORE method, non-volatility mode, STORE/RESTORE energy, area and silicon verification. All the simulations are completed with the same temperature $\left(27^{\circ} \mathrm{C}\right)$, and supply voltage $(2 \mathrm{~V})$.

$4 T 2 R$ nvSRAM cell [28] presents a real-time non- volatility with both WRITE and STORE executed simultaneously. This cell follows the differential sensing approach as

Table 1 NVSRAM cells original CMOS and ReRAM technology

\begin{tabular}{|c|c|c|c|c|c|}
\hline & $4 \mathrm{~T} 2 \mathrm{R}[28]$ & 7T1R [29] & $8 T 2 R[30]$ & 7T2R [31] & $8 \mathrm{~T} 2 \mathrm{R}[32]$ \\
\hline CMOS Technology & $90 \mathrm{~nm}$ & $32 \mathrm{~nm}$ & $22 \mathrm{~nm}$ FDSOI & $0.18 \mu \mathrm{m}$ TSMC & $0.18 \mu \mathrm{m}$ TSMC \\
\hline ReRAM Technology & $3 \mathrm{~nm}$ thick $\mathrm{HfOx}$ & $10 \times 10 \mathrm{~nm}^{2} \mathrm{Hf} / \mathrm{HfOx}$ & $10 \mathrm{~nm} \mathrm{HfO} 2$ & $\mathrm{HfOx}$ & ITRI BEOL Hf2O \\
\hline ReRAM Model & Spice Model [33] & unknown & Spice Model [34] & unknown & unknown \\
\hline
\end{tabular}

Table 2 Comparison between NVSRAM designs in term of different parameters

\begin{tabular}{llllll}
\hline & 4T2R [28] & 7T1R [29] & 8T2R [30] & 7T2R [31] & 8T2R [32] \\
\hline STORE Time & $1.45 \mathrm{~ns}$ & $0.24 \mathrm{~ns}$ & $0.24 \mathrm{~ns}$ & $1 \mathrm{~ns}$ & $0.87 \mathrm{~ns}$ \\
RESTORE Time & $20 \mathrm{ps}$ & $0.22 \mathrm{~ns}$ & $0.22 \mathrm{~ns}$ & $0.37 \mathrm{~ns}$ & $0.36 \mathrm{~ns}$ \\
RESTORE Method & Differential & Single-ended & Differential & Differential & Differential \\
Non- Volatility & Real- time & Before Shutdown & Before Shutdown & Before Shutdown & Before Shutdown \\
STORE Energy & $0.49 \mathrm{~nJ}$ & $30 \mathrm{pJ}$ & $50 \mathrm{pJ}$ & $90 \mathrm{pJ}$ & $87 \mathrm{pJ}$ \\
RESTORE Energy & $30 \mathrm{fJ}$ & $50 \mathrm{fJ}$ & $80 \mathrm{fJ}$ & $25 \mathrm{fJ}$ & $52 \mathrm{fJ}$ \\
Forming Energy & $1.7 \mathrm{~nJ}$ & $0.35 \mathrm{~nJ}$ & $0.77 \mathrm{~nJ}$ & $1 \mathrm{~nJ}$ & $6.345 \mathrm{~nJ}$ \\
Area & Small & Medium & Large & Medium & Large \\
Silicon Verified & No & No & No & Yes & Yes \\
\hline
\end{tabular}




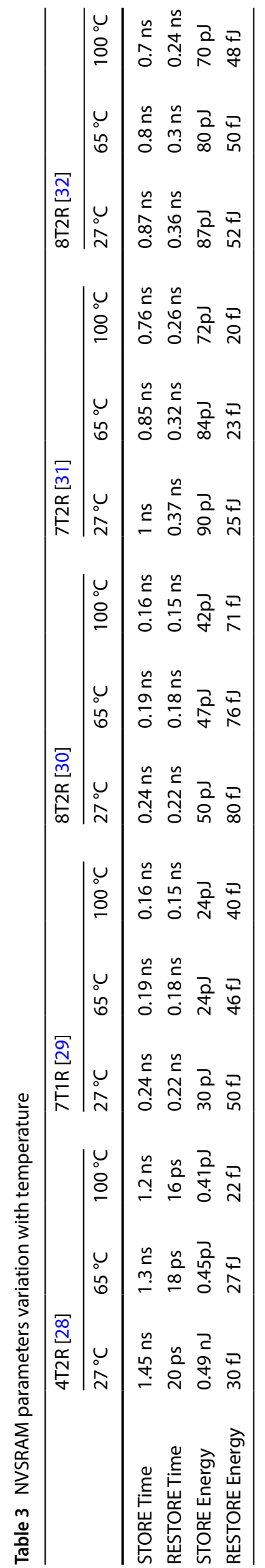

RESTORE method (2 OxRAMs). The structure of this design has a low area overhead, since the OxRAM devices replace the pMOS transistors. STORE/RESTORE times measured for this cell are $1.45 \mathrm{~ns}$ and 20 ps respectively. This cell is slower than conventional 6T SRAM due to programming of the OxRAM cell which occurs during each WRITE cycle. On the other hand, this design offers a real time non-volatility, and not a last-bit (or power-down) non-volatility as a trade-off for speed drawback. Also, this structure suffers from high power consumption as a STORE operation comes with a WRITE operation. STORE energy including SET and RESET operations is $0.49 \mathrm{~nJ}$. RESTORE energy is around $30 \mathrm{fJ}$. The energy dissipated by the OxRAM device during Forming is $1.7 \mathrm{~nJ}$.

7T1R [29] and 8T2R [30] nvSRAM cells are based on 6T SRAM core unlike 4T2R cell [18]. Although 7T1R cell [29] suffers from a slight increase of the variability in resistance and process, but this issue does not corrupt the normal operation of the nvSRAM design. The advantage of 7T1R cell [29] is its low power consumption and area, knowing that only one OxRAM is used. The STORE energy is $30 \mathrm{pJ}$ [29] which is slightly less than [30] with $50 \mathrm{pJ}$. Forming energy for the 7T1R cell is approximately half of that for $8 \mathrm{~T} 2 \mathrm{R}$.

Both structures scored similar numbers for RESTORE energy ( 50 and $80 \mathrm{fJ}$ ). Regarding STORE/RESTORE times, they follow the same scheme with $0.24 \mathrm{~ns}$ and $0.22 \mathrm{~ns}$ respectively. The asymmetric design (single-ended sensing as RESTORE method) makes the structure more susceptible to READ disturb, but it is not considered a significant drawback. $8 \mathrm{~T} 2 \mathrm{R}$ cell [30] is more consistent in the READ operation, but at the expanse of more occupied area and power consumption.

7T2R [31] and 8T2R [32] non-volatile SRAMs have achieved fast bit-to-bit parallel STORE/RESTORE operations ( $1 \mathrm{~ns}$ and $0.37 \mathrm{~ns}$ respectively), low energy requirements, and low READ/WRITE VDD enabling mobile chips to achieve low active-mode power consumption. Furthermore, low-energy STORE/RESTORE operation ( $90 \mathrm{pJ}$ $\& 25 \mathrm{fJ}$ for [31], and $0.87 \mathrm{~nJ} \& 52 \mathrm{fJ}$ for [32]) prevents data loss in mobile devices resulting from rapid power failure. Forming energy for [31] is $1 \mathrm{~nJ}$ and $6.345 \mathrm{~nJ}$ for [32].

Table 3 presents nvSRAM parameters changes with the temperature, including STORE/RESTORE time and energy. The STORE/RESTORE time and energy have decreased with the increase of the temperature. This time decrease is due to the effect of rising the temperature in accelerating the formation of the conductive filament in the switching medium [35]. As the energy has a linear relation with the time, any time change has a direct effect on the energy. Therefore, STORE energy for all cells have decreased. As for RESTORE energy, the variation was minimal since the 
RESTORE operation does not include a switching in the resistive state of the OXRAM device [36, 37].

Finally, it should be noted that the STORE time of the OxRAM devices is not constant as it depends on the programming conditions which are different for each memory cell. The same applies for the Forming time.

\section{Conclusion}

SRAM memory cells based on OxRAM are proposed as an enhanced structure to boost SRAM performances in terms of power consumption. In this context, the $1 \mathrm{~T} 1 \mathrm{R}$ OxRAM structure is used as a basic cell in different nvSRAM topologies, offering a large band of benefits while keeping a low design complexity. nvSRAM architectures have been explored, showing their advantages and drawbacks. As a conclusion, it appears that 4T2R nvSRAM cell offers real time non-volatility with low area with more power consumption compared to other nvSRAM architectures. The other architectures present a last-bit non-volatility with low power consumption.

Acknowledgment I would like to acknowledge the support of the Lebanese International University, Aix-Marseille University, and the support of Campus France (Eiffel scholarship).

Funding This study was funded by the Lebanese International University, Aix-Marseille University, and Campus France.

\section{Compliance with ethical standards}

Conflict of interest The authors declare that they have no conflict of interest.

\section{References}

1. Meena JS et al (2014) Overview of emerging nonvolatile memory technologies. Nanoscale Res Lett 9:526

2. Hamdioui S et al (2014) Memristor based memories: technology, design and test. In: 9th IEEE international conference on design and technology of integrated systems in nanoscale era (DTIS)

3. lelmini $D$ (2016) Resistive switching memories based on metal oxides: mechanisms, reliability and scaling. Semicond Sci Technol 31(6):063002

4. Garbin D et al (2015) HfO2-based OxRAM devices as synapses for convolutional neural networks. IEEE Trans Electron Devices 62(8):2494-2501

5. Bocquet $M$ et al (2014) Robust compact model for bipolar oxidebased resistive switching memories. IEEE Trans Electron Devices 61(3):674-681

6. Bazzi $\mathrm{H}$ et al (2018) Design of hybrid CMOS non-volatile SRAM cells in $130 \mathrm{~nm}$ RRAM technology. In: 2018 30th international conference on microelectronics (ICM), Sousse, Tunisia, pp 228-231
7. Xie Y (2014) Emerging memory technologies: design, architecture, and applications. Springer, New York

8. Chang M et al (2014) Challenges at circuit designs for resistivetype Nonvolatile memory and nonvolatile logics in mobile and cloud applications. In: 2014 12th IEEE international conference on solid-state and integrated circuit technology (ICSICT), Guilin, pp 1-4

9. Sterpone L et al (2011) A new reconfigurable clock-gating technique for low power SRAM-based FPGAs. In: 2011 design, automation and test in Europe, Grenoble, pp 1-6

10. Singh $P$ et al (2017) Ultra-low power, process-tolerant 10T (PT10T) SRAM with improved read/write ability for internet of things (IoT) applications. J. Low Power Electron Appl 7:24

11. Dou C et al (2017) Challenges of emerging memory and memristor based circuits: nonvolatile logics, loT security, deep learning and neuromorphic computing. In: 2017 IEEE 12th international conference on ASIC (ASICON)

12. Portal J-M et al (2014) An overview of non-volatile flip-flops based on emerging memory technologies. J Electron Sci Technol 12(2):173-181

13. Bard AJ, Faulkner LR (2001) Electrochemical methods: fundamentals and applications. Wiley, New York

14. Fackenthal R et al (2014) 19.7 A 16 Gb ReRAM with $200 \mathrm{MB} / \mathrm{s}$ write and $1 \mathrm{~GB} / \mathrm{s}$ read in $27 \mathrm{~nm}$ technology. In: 2014 IEEE international solid-state circuits conference digest of technical papers (ISSCC)

15. Molas $\mathrm{G}$ et al (2018) Resistive memories (RRAM) variability: challenges and solutions. ECS Trans 86(3):35-47

16. Bocquet M, Deleruyelle D, Aziza H, Muller C, Portal J (2013) Compact modeling solutions for OxRAM memories. In: 2013 IEEE Faible Tension Faible Consommation, Paris, pp 1-4

17. Aziza $\mathrm{H}$ et al (2018) Resistive RAMs as analog trimming elements. Solid-State Electron 142:52-55

18. Canet $P$ et al (2016) Impact of resistive paths on NVM array reliability: application to Flash \& ReRAM memories. Microelectron Reliab 64:36-41

19. Nardi $F$ et al (2012) Resistive switching by voltage-driven ion migration in bipolar RRAM part I: experimental study. IEEE Trans Electron Devices 59(9):2461-2467

20. DeSalvo B et al (2015) Emerging resistive memories for low power embedded applications and neuromorphic systems. In: 2015 IEEE international symposium on circuits and systems (ISCAS), Lisbon, pp 3088-3091

21. Dimin $\mathrm{N}$ et al (2010) Impact of process variations on emerging memristor. In: Proceedings of the 47th design automation conference (DAC), 2010 ACM/IEEE, IEEE, pp 877-882

22. Walczyk $C$ et al (2011) Impact of temperature on the resistive switching behavior of embedded HfO2-based RRAM devices. IEEE Trans Electron Dev 58:3124-3131

23. Jiang $Z$ et al (2009) Characterization of line edge roughness and line width roughness of nano-scale typical structures. In: Proceedings of 4th IEEE NEMS, pp 299-303

24. Bocquet $\mathrm{M}$ et al (2018) In-memory and error-immune differential RRAM implementation of binarized deep neural networks. In: IEDM Tech. Dig., San Francisco USA, pp 20.6.1-20.6.4

25. Wei $Z$ et al (2011) Demonstration of high-density ReRAM ensuring 10-year retention at $85^{\circ} \mathrm{C}$ based on a newly developed reliability model. In: 2011 international electron devices meeting

26. Azzaz M et al (2016) Endurance/retention trade off in $\mathrm{HfOx}$ and TaOx based RRAM. In: 2016 IEEE 8th international memory workshop (IMW)

27. Hajri B et al (2017) Oxide-based RRAM models for circuit designers: a comparative analysis. In 12th international conference on design and technology of integrated systems in nanoscale era (DTIS), Palma de Mallorca, pp 1-6 
28. Majumdar S et al (2016) Hybrid CMOS-OxRAM based 4T-2R NVSRAM with efficient programming scheme. In: 2016 16th nonvolatile memory technology symposium (NVMTS), Pittsburgh, PA, pp 1-4

29. Wei W et al (2014) Design of a nonvolatile 7T1R SRAM cell for instant-on operation. IEEE Trans Nanotechnol 13(5):905-916

30. Turkyilmaz O et al (2012) RRAM-based FPGA for "normally off, instantly on" applications. In: 2012 IEEE/ACM international symposium on nanoscale architectures, Amsterdam, pp 101-108

31. Sheu $S$ et al (2013) A ReRAM integrated 7T2R non-volatile SRAM for normally-off computing application. In: 2013 IEEE Asian solid-state circuits conference (A-SSCC), Singapore, pp 245-248

32. Chiu $P$ et al (2012) Low store energy, low VDDmin, 8T2R nonvolatile latch and SRAM with vertical-stacked resistive memory (Memristor) devices for low power mobile applications. IEEE J Solid State Circuits 47(6):1483-1496
33. Li H et al (2015) Variation-aware, reliability-emphasized design and optimization of RRAM using SPICE model. In: Design, automation and test in Europe conference and exhibition (DATE), Grenoble, pp 1425-1430

34. Onkaraiah S et al (2012) Bipolar ReRAM based non-volatile flipflops for low-power architectures. In: NEWCAS, pp 1-4

35. Lv H et al (2015) Evolution of conductive filament and its impact on reliability issues in oxide-electrolyte based resistive random access memory. Sci Rep 5:7764

36. Bazzi $\mathrm{H}$ et al (2018) Novel RRAM CMOS non-volatile memory cells in $130 \mathrm{~nm}$ technology. In: ICCA, Beirut, pp 390-393

37. Bazzi H et al (2020) RRAM-based non-volatile SRAM cell architectures for ultra-low-power applications. Analog Integr Circ Sig Process

Publisher's Note Springer Nature remains neutral with regard to jurisdictional claims in published maps and institutional affiliations. 